\title{
Global Sagittal Alignment and Clinical Outcomes after 1-3 Short-Segment Lumbar Fusion in Degenerative Spinal Diseases
}

\author{
Yung-Hun Youn, Kyu-Jung Cho, Yeop Na, Jeong-Seok Kim \\ Department of Orthopaedic Surgery, Inha University Hospital, Incheon, Korea
}

\begin{abstract}
Study Design: Level III retrospective study.
Purpose: We investigated the impact of short-segment lumbar fusion on the restoration of global sagittal alignment and the correlations between spino-pelvic parameters and clinical outcomes.

Overview of Literature: Sagittal imbalance leads to energy consumption and pain in maintaining a standing position. For adult spinal deformity, it is critical to create optimal lumbar lordosis (LL) in order to achieve restoration of sagittal imbalance. However, surgeons do not pay attention to correcting $L L$ in short-segment lumbar fusion.

Methods: A total of 69 patients with transforaminal lumbar interbody fusion (TLIF) for degenerative spinal disease were evaluated with a minimum 2-year follow-up. All patients underwent TLIF with hyper-lordotic angle cages to achieve higher LL. Radiological spino-pelvic parameters including sagittal vertical axis (SVA) and clinical outcomes using the Oswestry Disability Index (ODI) and Numeric Rating Scale (NRS) were evaluated.

Results: The average $L L$ was $35.8^{\circ} \pm 9.9^{\circ}$ before surgery, $42.3^{\circ} \pm 9.3^{\circ} 1$ year after surgery, and $40.3^{\circ} \pm 10.2^{\circ} 2$ years after surgery $(p<0.01)$. The average SVA was $43.1 \pm 6.2 \mathrm{~mm}$ before surgery, $21.2 \pm 4.9 \mathrm{~mm} 1$ year after surgery, and $34.0 \pm 4.7 \mathrm{~mm} 2$ years after surgery $(p<0.01)$. The average $L L$ and SVA improved in two- or three-segment fusion, but not in one-segment fusion. The correlation between $\triangle L L$ and $\triangle S V A$ was significant in all segment fusions. The correlation between $\triangle L L$ and $\triangle S V A$ was more significant at the $L 4-5$ and $L 5-S 1$ segments than at L3-4. ODI was significantly correlated with SVA $(p<0.05)$. NRS showed no correlation with the radiological parameters. Conclusions: Two- or three-segment lumbar fusion using hyper-lordotic angle cages improved LL and SVA. A significant correlation between the correction of $L L$ and SVA was found. Higher correction of $L L$ using hyper-lordotic angle cages is thus recommended in short-segment lumbar fusion, since postoperative improvements of SVA significantly affect clinical outcomes.
\end{abstract}

Keywords: Lumbar lordosis; Sagittal vertical axis; Sagittal balance; Spino-pelvic parameter; Clinical outcome; Hyper-lordotic cage

\section{Introduction}

Balanced spinal alignment allows an individual to achieve a standing posture with minimal musculoskeletal energy.
In patients with spinal deformity, achieving sagittal balance is essential to obtain good clinical outcomes. For the optimal correction of sagittal alignment, restoring a sagittal vertical axis (SVA) $<5 \mathrm{~cm}$ is suggested [1-3]. Spino-pel-

\footnotetext{
Received May 6, 2021; Revised Jun 30, 2021; Accepted Jul 6, 2021

Corresponding author: Kyu-Jung Cho

Department of Orthopedic Surgery, Inha University Hospital, 27 Inhang-ro, Jung-gu, Incheon 22332, Korea

Tel: +82-32-890-3661, Fax: +82-32-890-2619, E-mail: chokj@inha.ac.kr
} 
vic parameters such as SVA and pelvic tilt (PT) are known to be related to clinical outcomes after spinal fusion surgery. SVA is a good indicator of health-related quality of life (HRQOL). PT is related to compensation for the loss of lumbar lordosis (LL) and correlated with postoperative residual pain and disability [4].

Insufficient correction of LL might cause iatrogenic flat-back deformity after short-segment as well as longsegment fusions $[5,6]$. Even when LL is insufficiently corrected in short-segment lumbar fusion, compensatory hyperextension at the unfused segments and pelvic retroversion can prevent global sagittal imbalance just after surgery. However, subsequent disk degeneration at the unfused segment or adjacent segment disease can eventually cause global sagittal imbalance [7].

There is still controversy over whether short-segment lumbar fusion may affect global sagittal alignment. Cho et al. [8] demonstrated that global sagittal alignment improved through short-segment fusion in patients with spinal stenosis and spondylolisthesis. In addition, Alqroom [9] reported similar findings, namely, that short-segment fusion surgery improved spino-pelvic parameters. In contrast, another study reported that short lumbosacral fixation did not significantly restore sagittal balance or change sagittal spino-pelvic alignment [10]. Further spinal stenosis, the restoration of SVA turned out to differ in a manner dependent on the number of fused segments and the level of the fusion segment $[8,9]$.

Against this background, the purposes of the current study were (1) to evaluate whether short-segment fusion for degenerative spinal diseases improved global spinal alignment and (2) to determine the change of radiological spino-pelvic parameters according to the fusion level and number of fused segments. Which radiological parameter among SVA and PT was more closely related to the improvement of clinical outcomes was also investigated.

\section{Materials and Methods}

\section{Patient selection}

From January 2012 to January 2017, 290 patients who underwent posterior instrumentation with additional transforaminal lumbar interbody fusion (TLIF) for degenerative spinal disease in a single hospital were evaluated retrospectively with a minimum 2-year follow-up. Among these patients, 69 consecutive patients with one- to three-segment lumbar fusion were included in this study. Only patients with TLIF performed at all segments were included. For example, patients who underwent posterior fusion at two segments and TLIF at one segment were not included. Patients who missed follow-ups, had insufficient radiological and clinical data, or experienced postoperative complications such as adjacent segment disease, junctional problem, cage subsidence, pseudarthrosis, or implant failure were excluded. All patients underwent the same surgical procedures, decompression and a combination of TLIF and posterolateral inter-transverse fusion. The Institutional Review Board (IRB) approved this study (INHAUH 2019-06-023). This study was approved by the IRB for the exemption of consent for the patient. Informed consent was not obtained from the patients because of a retrospective study.

\section{Radiological and clinical assessment}

The radiological protocol consisted of standing anteriorposterior and lateral radiographs of the whole spine for the spinal imbalance in all patients. The follow-up protocol included radiologic estimation before operation at the 1-year follow-up and the following measurements at the 2-year follow-up: (1) LL (the angle between tangent lines to the superior endplate of $\mathrm{L} 1$ and superior endplate of $\mathrm{S} 1$ ); (2) SVA (the distance between the C7 plumb line and the postero-superior corner of the sacrum); (3) pelvic incidence (PI; the angle between the perpendicular line of the sacral endplate and the line joining the middle of the sacral endplate and the hip center); (4) PT (angle between a vertical line and the line from the middle of the sacral endplate to the hip center); (5) sacral slope (SS; the angle between the sacral endplate and a horizontal line); and (6) PI-LL. The Oswestry Disability Index (ODI) and Numeric Rating Scale (NRS) were also checked to evaluate the correlations with the spino-pelvic parameters SVA, PT, and PI-LL.

\section{Surgical technique}

A senior spine surgeon performed posterior decompression and pedicle screw instrumented lumbar fusion in all patients. To achieve greater correction of $\mathrm{LL}$, a $15^{\circ}$-angled hyper-lordotic cage was inserted. Furthermore, after pedicle screws and rods were in place, pedicle screws were compressed along bended rods. 


\section{Statistical analysis}

All of the patients' characteristics were compared through chi-square test and Student $t$-test. The variation of spinopelvic parameters was calculated using a paired $t$-test and one-way analysis of variance. The correlation between $\Delta \mathrm{LL}$ (postoperative LL-preoperative LL) and $\triangle \mathrm{SVA}$ (preoperative SVA-postoperative SVA) was analyzed using Pearson's correlation analysis. Further statistical analyses were performed to evaluate the correlations between the clinical outcome and the spino-pelvic parameters through Mann-Whitney $U$-test and Pearson's correlation analysis. All statistical results are presented as mean \pm standard deviation, and statistical significance is indicated as a $p$-value $<0.05$. SPSS Statistics for Windows ver. 10.0 (SPSS Inc., Chicago, IL, USA) was used for the statistical analysis.

\section{Results}

\section{Patients' demographics}

A total of 69 patients who underwent TLIF with hyperlordotic cages and pedicle screw instrumentation were included in this study. Their demographic characteristics are summarized in Table 1. The diagnosis was degenerative spinal stenosis in 40 patients, degenerative spondylolisthesis in 20 patients, spondylolytic spondylolisthesis in eight patients, and adult degenerative lumbar scoliosis in one patient. The mean age was $64.5 \pm 9.1$ years (range, 22-85 years), with the patients consisting of 41 males and 28 females. The average number of fused segments was $1.79 \pm 0.86$ (range, 1 to 3 segments), including onesegment fusion in 30 patients, two-segment fusion in 23 patients, and three-segment fusion in 16 patients. The lumbar fusion was mostly performed at the L3-4, L4-5, and L5-S1 levels, including at L3-4 in 35 patients, L4-5 in 56 patients, L5-S1 in 27 patients, and L2-3 in six patients.

\section{Radiological outcomes}

1) LL

The average LL was $35.8^{\circ} \pm 9.9^{\circ}$ before surgery, $42.3^{\circ} \pm 9.3^{\circ}$

Table 1. Patients' demographics ( $n=69)$

\begin{tabular}{lc}
\hline Characteristic & Value \\
\hline Age (yr) & $64.5 \pm 9.1$ (22-85) \\
\hline Sex & \\
\hline Male & 41 \\
\hline Female & 28 \\
\hline Follow-up periods (yr) & $2.8 \pm 1.5(2-5)$ \\
\hline No. of fused segment (levels) & \\
\hline 1-Segment & 30 \\
\hline L34 & 3 \\
\hline L45 & 18 \\
\hline L5S1 & 9 \\
\hline 2-Segment & 23 \\
\hline L234 & 1 \\
\hline L345 & 15 \\
\hline L45S1 & 7 \\
\hline 3-Segment & 16 \\
\hline L2345 & 5 \\
\hline L345S1 & 11 \\
\hline Preoperative diagnosis (\%) & 20 \\
\hline Spinal stenosis & 8 \\
\hline Degenerative spondylolithesis & 1 \\
\hline Spondylolytic spondylolithesis & \\
\hline Adult degenerative lumbar scoliosis & \\
\hline & \\
\hline
\end{tabular}

Values are presented as mean \pm standard deviation (range) or number of patients.

Table 2. Correction of $L L$ after 1-3-segment fusions

\begin{tabular}{|c|c|c|c|c|c|}
\hline \multirow{2}{*}{$\operatorname{LL}\left({ }^{\circ}\right)$} & \multirow{2}{*}{ Preoperative } & \multicolumn{2}{|c|}{ P0 $1 \mathrm{yr}$} & \multicolumn{2}{|c|}{ PO 2 yr } \\
\hline & & Mean \pm SD & $p$-value & Mean $\pm S D$ & $p$-value \\
\hline Average & $35.8 \pm 9.9$ & $42.3 \pm 9.3$ & $<0.01^{*}$ & $40.3 \pm 10.2$ & $<0.01^{*}$ \\
\hline 1-Segment $(n=30)$ & $41.1 \pm 11.7$ & $45.0 \pm 7.1$ & 0.26 & $44.2 \pm 8.7$ & 0.09 \\
\hline 2-Segments ( $\mathrm{n}=23)$ & $35.6 \pm 10.9$ & $42.1 \pm 10.7$ & $<0.01^{*}$ & $40.6 \pm 10.1$ & $<0.01^{*}$ \\
\hline 3-Segments $(\mathrm{n}=16)$ & $26.4 \pm 8.7$ & $37.5 \pm 7.9$ & $<0.01^{*}$ & $32.4 \pm 8.4$ & $0.02^{*}$ \\
\hline
\end{tabular}

Values are presented as mean $\pm S D$, unless otherwise stated.

LL, lumbar lordosis; PO, postoperative; SD, standard deviation.

${ }^{*} p<0.05$; statistical significance. 
Table 3. Restoration of SVA after 1-3-segment fusions

\begin{tabular}{|c|c|c|c|c|c|}
\hline \multirow{2}{*}{ SVA (mm) } & \multirow{2}{*}{ Preoperative } & \multicolumn{2}{|c|}{ PO 1 yr } & \multicolumn{2}{|c|}{$\mathrm{PO} 2 \mathrm{yr}$} \\
\hline & & Mean \pm SD & $p$-value & Mean \pm SD & $p$-value \\
\hline Average & $43.1 \pm 6.2$ & $21.2 \pm 4.9$ & $<0.01^{*}$ & $34.0 \pm 4.7$ & $<0.01^{*}$ \\
\hline 1-Segment $(n=30)$ & $33.5 \pm 4.9$ & $15.6 \pm 5.8$ & 0.15 & $22.9 \pm 3.3$ & 0.14 \\
\hline 2-Segments (n=23) & $37.5 \pm 6.8$ & $13.5 \pm 4.0$ & $0.04^{*}$ & $26.4 \pm 4.4$ & 0.18 \\
\hline 3-Segments ( $n=16)$ & $69.2 \pm 4.2$ & $42.8 \pm 6.4$ & $0.02^{*}$ & $65.6 \pm 5.6$ & $0.03^{*}$ \\
\hline
\end{tabular}

Values are presented as mean $\pm S D$, unless otherwise stated.

SVA, sagittal vertical axis: PO, postoperative; SD, standard deviation.

${ }^{*} p<0.05$; statistical significance.

1 year after surgery $(p<0.01)$, and $40.3^{\circ} \pm 10.2^{\circ} 2$ years after surgery $(p<0.01)$. Although the correction of LL after one-segment fusion was not statistically significant, the improvement of LL after two-segment and three-segment fusions was statistically significant (Table 2).

Fusion surgery was performed at L3-4 in 35 patients, L4-5 in 56 patients, and L5-S1 in 27 patients. The average LL in the L3-4 fusion group was $16.5^{\circ} \pm 7.9^{\circ}$ before surgery, $23.6^{\circ} \pm 6.8^{\circ} 1$ year after surgery $(p=0.04)$, and $23.0^{\circ} \pm 9.2^{\circ} 2$ years after surgery $(p=0.05)$. The average LL in the L4 -5 fusion group was $22.9^{\circ} \pm 14.0^{\circ}$ before surgery, $32.1^{\circ} \pm 10.1^{\circ} 1$ year after surgery $(p<0.01)$, and $31.4^{\circ} \pm 10.0^{\circ}$ 2 years after surgery $(p<0.01)$. The average LL in the L5S1 fusion group was $18.2^{\circ} \pm 11.8^{\circ}$ before surgery, $28.7^{\circ} \pm 9.5^{\circ}$ 1 year after surgery $(p<0.01)$, and $29.1^{\circ} \pm 9.8^{\circ} 2$ years after surgery $(p<0.01)$.

\section{2) SVA}

The average SVA changed from $43.1 \pm 6.2 \mathrm{~mm}$ before surgery to $21.2 \pm 4.9 \mathrm{~mm} 1$ year after surgery $(p<0.01)$ and $34.0 \pm 4.7 \mathrm{~mm} 2$ years after surgery $(p<0.01)$. The change of SVA after one-segment fusion was not statistically significant $(p>0.05)$. On the other hand, SVA improved significantly after two-segment and three-segment fusion. With two-segment fusion, it was $37.5 \pm 6.8 \mathrm{~mm}$ before surgery, $13.5 \pm 4.0 \mathrm{~mm} 1$ year after surgery $(p=0.04)$, and $26.4 \pm 4.4$ $\mathrm{mm} 2$ years after surgery $(p=0.18)$. With three-segment fusion, it was restored from $69.2 \pm 4.2 \mathrm{~mm}$ before surgery to $42.8 \pm 6.4 \mathrm{~mm} 1$ year after surgery $(p=0.02)$ and $65.6 \pm 5.6$ $\mathrm{mm} 2$ years after surgery $(p=0.03)$. During the follow-up period, it was observed that the restoration of SVA had gradually decreased over time after surgery (Table 3 ).

The average SVA in the L3-4 fusion group was $25.6 \pm 9.6$ $\mathrm{mm}$ before surgery, $10.0 \pm 8.8 \mathrm{~mm} 1$ year after surgery
Table 4. Correlations between $\triangle L L$ and $\triangle S V A$ according to the number of fused segments

\begin{tabular}{lccccc} 
Variable & Category & $\Delta L L\left({ }^{\circ}\right)$ & $\Delta S V A(m m)$ & $p$-value & $C C$ \\
\hline Average & P0 1 yr-preop & $6.5 \pm 6.2$ & $21.9 \pm 5.8$ & $0.01^{*}$ & 0.402 \\
& P0 2 yr-preop & $4.5 \pm 5.8$ & $11.2 \pm 5.9$ & $0.01^{*}$ & 0.383 \\
\hline 1-Segment (n=30) & P0 1 yr-preop & $3.9 \pm 6.4$ & $17.9 \pm 7.8$ & $0.05^{*}$ & 0.446 \\
& P0 2 yr-preop & $3.1 \pm 7.1$ & $10.6 \pm 6.6$ & $0.02^{*}$ & 0.405 \\
\hline 2-Segment (n=23) & P0 1 yr-preop & $6.5 \pm 9.1$ & $24.0 \pm 5.7$ & $0.02^{*}$ & 0.492 \\
\hline & P0 2 yr-preop & $5.0 \pm 9.4$ & $11.1 \pm 6.9$ & $0.01^{*}$ & 0.424 \\
\hline 3-Segment (n=16) & P0 1 yr-preop & $11.1 \pm 6.9$ & $26.4 \pm 7.2$ & $0.01^{*}$ & 0.562 \\
\hline & P0 2 yr-preop & $6.0 \pm 10.8$ & $12.5 \pm 5.7$ & $0.04^{*}$ & 0.392 \\
\hline
\end{tabular}

Values are presented as mean \pm standard deviation, unless otherwise stated. LL, lumbar lordosis; SVA, sagittal vertical axis; $\triangle \mathrm{LL}$, postoperative LL-preoperative LL; $\triangle S V A$, preoperative SVA-postoperative SVA; CC, correlation coefficient; PO, postoperative; Preop, preoperative.

${ }^{*} p<0.05$; statistical significance.

$(p=0.04)$, and $14.8 \pm 3.9 \mathrm{~mm} 2$ years after surgery $(p=0.05)$. The average SVA in the L4 -5 fusion group was $30.1 \pm 9.6$ $\mathrm{mm}$ before surgery, $9.4 \pm 8.2 \mathrm{~mm} 1$ year after surgery $(p<0.01)$, and $11.2 \pm 7.9 \mathrm{~mm} 2$ years after surgery $(p=0.04)$. The average SVA in the L5-S1 fusion group was $67.5 \pm 7.9$ $\mathrm{mm}$ before surgery, $39.0 \pm 9.8 \mathrm{~mm} 1$ year after surgery $(p<0.01)$, and $43.2 \pm 6.5 \mathrm{~mm} 2$ years after surgery $(p<0.01)$.

\section{3) Correlations between $\triangle \mathrm{LL}$ and $\triangle \mathrm{SVA}$}

The corrections of LL and SVA were $5.3^{\circ} \pm 6.0^{\circ}$ and $24.3 \pm 6.8$ $\mathrm{mm} 1$ year after surgery and $4.4^{\circ} \pm 5.8^{\circ}$ and $17.1 \pm 5.9 \mathrm{~mm}$ 2 years after surgery, respectively. Pearson's correlation analysis was conducted to confirm the linearity of the correlation between $\triangle \mathrm{LL}$ (postoperative LL-preoperative LL) and $\triangle$ SVA (preoperative SVA-postoperative SVA). There was a significant correlation between these variables both 1 year $(p=0.01$, correlation coefficient $=0.397)$ and 2 years 
Table 5. Correlations between $\triangle L L$ and $\triangle S V A$ according to the $L 3-4, L 4-5$, and L5-S1-segment fusion

\begin{tabular}{lccccc} 
Variable & Category & $\Delta \mathrm{LL}\left({ }^{\circ}\right)$ & $\Delta S V A(m m)$ & $p$-value & CC \\
L3-4 & P0 1 yr-preop & $7.1 \pm 8.6$ & $15.6 \pm 6.4$ & $0.04^{*}$ & 0.062 \\
& P0 2 yr-preop & $6.5 \pm 9.9$ & $10.8 \pm 8.9$ & $0.05^{*}$ & 0.043 \\
\hline \multirow{2}{*}{ 4-5 } & P0 1 yr-preop & $9.2 \pm 9.3$ & $20.7 \pm 7.4$ & $<0.01^{*}$ & 0.123 \\
\hline \multirow{2}{*}{ L5-S1 } & P0 2 yr-preop & $8.5 \pm 10.7$ & $18.9 \pm 6.9$ & $<0.01^{*}$ & 0.104 \\
& P0 1 yr-preop & $10.5 \pm 10.5$ & $28.5 \pm 8.2$ & $<0.01^{*}$ & 0.194 \\
\hline
\end{tabular}

Values are presented as mean \pm standard deviation, unless otherwise stated. $\mathrm{LL}$, lumbar lordosis; SVA, sagittal vertical axis; $\Delta \mathrm{LL}$, postoperative $\mathrm{LL}-$ preoperative LL; $\triangle S V A$, preoperative SVA-postoperative SVA; CC, correlation coefficient; PO, postoperative; Preop, preoperative.

${ }^{*} p<0.05$; statistical significance.

after surgery $(p=0.01$, correlation coefficient $=0.353)$. The correlation between $\triangle \mathrm{LL}$ and $\triangle \mathrm{SVA}$ was more significant in two-segment and three-segment fusion than in onesegment fusion. The greater the number of fused lumbar segments, the more significant the correlation between $\triangle \mathrm{LL}$ and $\triangle$ SVA (Table 4).

The analysis of the correlation between $\triangle \mathrm{LL}$ and $\triangle \mathrm{SVA}$ was conducted according to the L3-4, L4-5, or L5-S1 fusion groups, including L4-5 in 40 patients, L3-4 in 28 patients, and L5-S1 in 20 patients. The correlations between $\Delta \mathrm{LL}$ and $\triangle \mathrm{SVA}$ in the L4-5 and L5-S1 fusion groups were more significant than in the L3-4 fusion group, meaning that the correction of lordosis at L4-5 and L5-S1 more markedly affected the restoration of SVA than that at L3-4 (Table 5).

4) Other spino-pelvic parameters

The average PI was $47.8^{\circ} \pm 10.6^{\circ}$ before surgery, $47.1^{\circ} \pm 9.3^{\circ}$ 1 year after surgery $(p=0.50)$, and $48.0^{\circ} \pm 9.8^{\circ} 2$ years after surgery $(p=0.75)$. PI-LL was successfully corrected close to $10^{\circ}$ after surgery. The average PI-LL was $14.5^{\circ} \pm 13.8^{\circ}$ before surgery, $10.3^{\circ} \pm 11.2^{\circ} 1$ year after surgery $(p<0.01)$, and $10.5^{\circ} \pm 8.2^{\circ} 2$ years after surgery $(p=0.03)$.

The average PT did not change after the surgery; it was $17.5^{\circ} \pm 8.5^{\circ}$ before surgery, $17.71^{\circ} \pm 8.6^{\circ} 1$ year after surgery $(p=0.93)$, and $17.9^{\circ} \pm 8.4^{\circ} 2$ years after surgery $(p=0.69)$. With one-segment fusion, PT was $16.9^{\circ} \pm 9.4^{\circ}$ before surgery, $17.2^{\circ} \pm 10.5^{\circ} 1$ year after surgery $(p=0.62)$, and $17.7^{\circ} \pm 7.2^{\circ} 2$ years after surgery $(p=0.82)$. With two-segment fusion, it was $17.6^{\circ} \pm 8.1^{\circ}$ before surgery, $17.8^{\circ} \pm 7.6^{\circ} 1$ year after surgery $(p=0.26)$, and $17.3^{\circ} \pm 9.4^{\circ} 2$ years after surgery $(p=0.49)$. With three-segment fusion, it
Table 6. Correlation between spinopelvic parameters and clinical outcomes

\begin{tabular}{lcc} 
Score & $R$ & $p$-value \\
\hline ODI vs. SVA & 0.43 & $<0.05^{*}$ \\
\hline NRS vs. SVA & 0.39 & 0.28 \\
ODI vs. PT & 0.42 & 0.58 \\
NRS vs. PT & 0.35 & 0.69 \\
ODI vs. PI-LL & 0.23 & 0.35 \\
NRS vs. PI-LL & 0.19 & 0.41 \\
\hline
\end{tabular}

ODI, Oswestry Disability Index; SVA, sagittal vertical axis; NRS, Numeric Rating Scale; PT, pelvic tilt; PI, pelvic incidence; LL, lumbar lordosis.

${ }^{*} p<0.05$; statistical significance.

was changed from $19.4^{\circ} \pm 8.6^{\circ}$ before surgery to $20.2^{\circ} \pm 7.0^{\circ}$ 1 year after surgery $(p=0.50)$ and $20.9^{\circ} \pm 7.9^{\circ} 2$ years after surgery $(p=0.59)$.

\section{Clinical outcomes}

\section{1) ODI and NRS}

Most patients displayed improvements in terms of the ODI and NRS after surgery. The average ODI in the patients who had received one- to three-segment fusion was $53.3 \pm 8.9$ before surgery, $35.6 \pm 8.51$ year after surgery $(p<0.01)$, and $30.2 \pm 7.62$ years after surgery $(p<0.01)$. The average NRS on the back was $7.0 \pm 5.8$ before surgery, $5.5 \pm 5.91$ year after surgery $(p<0.01)$, and $4.6 \pm 6.12$ years after surgery $(p<0.01)$. The average NRS on the legs was $6.9 \pm 7.8$ before surgery, $4.1 \pm 7.61$ year after surgery $(p<0.01)$, and $3.6 \pm 5.82$ years after surgery $(p<0.01)$.

2) Correlations between clinical outcomes and spinopelvic parameters

The correlations between clinical outcomes (ODI and NRS) and radiological spino-pelvic parameters (SVA, PT, and PI-LL) were analyzed using Pearson's correlation test, showing that ODI was significantly correlated with SVA $(R=0.43, p<0.05)$. NRS was not correlated with SVA $(R=0.39, p>0.05)$. On the other hand, LL, PT, and PI-LL did not affect clinical outcomes (Table 6).

\section{Discussion}

Achieving optimal LL in spinal fusion surgery improves spino-pelvic alignment, resulting in good clinical outcomes [11-13]. Insufficient correction of LL and PI-LL mismatch may cause adjacent segment degeneration and 
subsequent sagittal imbalance [14-16]. Sagittal decompensation is particularly likely to develop in patients with preoperative severe sagittal imbalance or higher PI. Greater correction of LL is needed in these patients [17]. Certain radiological parameters provide the surgeon with a guide for restoring spino-pelvic balance [18]. PI-LL $=10^{\circ}$ sets the stage for the achievement of successful harmonious spino-pelvic realignment $[19,20]$, even though the sagittal spino-pelvic alignment varies with age. Lafage et al. [21] demonstrated that operative realignment targets should be $\mathrm{PI}-\mathrm{LL}=16.7^{\circ}$ for patients aged over 75 years.

Long-segment fusion is clearly more efficient for restoring SVA than short-segment fusion in adult spinal deformity. Lumbar fusion for degenerative spinal disease is much more likely to improve spino-pelvic parameters and sagittal alignment when it is performed on long segments than on short segments. In short-segment fusion surgery for degenerative lumbar spine with minimal sagittal imbalance, the correction of LL has not been emphasized. However, there is a growing awareness that the restoration of sagittal alignment is important even in short-segment fusion.

Controversy still remains over whether short-segment lumbar fusion affects the restoration of global sagittal alignment. Consensus has been reached that segmental lordosis is restored with short-segment lumbar fusion. However, the findings regarding the restoration of global sagittal alignment have differed depending on the report. For example, Korovessis et al. [10] found that short (one or two segments) lumbosacral fixation did not significantly change the sagittal spino-pelvic balance in adult patients with a preoperatively balanced spine. SVA decreased postoperatively but returned to the baseline value at the final observation. Alqroom [9] divided 56 patients who underwent lumbar fusion surgery into two groups: a one-level group and a two-level group. Patients in the onelevel group showed only small alterations in spino-pelvic parameters, while those in the two-level group achieved SVA. This study showed that the number of fused level with hyper-lordotic cage was associated with the overall correction of sagittal imbalance. The correlation between $\triangle \mathrm{LL}$ and $\triangle \mathrm{SVA}$ was more significant in two- to threesegment fusion than in one-segment fusion.

Spinal stenosis and resultant sagittal imbalance are difficult to differentiate from the sagittal imbalance due to adult spinal deformity. In radiographs, it has been reported that the PT/PI ratio can differ between the two dif- ferent sagittal balances [22]. Pelvic retroversion can be observed in both spinal stenosis and adult spinal deformity, but the ratio is expected not to be the same. A significant improvement in the SVA may occur in spinal stenosis patients merely due to decompression surgery as the compensatory crouch disappears. PT represents a compensatory mechanism by pelvic retroversion for the sagittal imbalance. In our study, average PT was not changed after surgery, being $17.5^{\circ} \pm 8.5^{\circ}$ before surgery, $17.7^{\circ} \pm 8.6^{\circ} 1$ year after surgery $\left(p=0.93\right.$ ), and $17.9^{\circ} \pm 8.4^{\circ} 2$ years after surgery $(p=0.69)$, indicating that the improvement of SVA was not related to pelvic compensation. Therefore, it can be concluded that the hyper-lordotic TLIF cage contributed to the improvement in the SVA.

The improvement of SVA and LL decreased at the 2-year follow-up compared with that at 1 year. The deterioration of radiological parameters might be associated with two factors. The first factor is loss of correction (LOC) in the disk space between the vertebral body and the cages. To obtain more LL restoration, the screws were tightened on the rod with posterior compression between two screw heads. This procedure creates a space between the endplate and the cages, which eventually narrows with weightbearing. The second factor is subsequent disc degeneration at the unfused segments. Considering this LOC during follow-up periods, it is advantageous to restore as much of $\mathrm{LL}$ as possible.

Although improving sagittal alignment is important in short-segment fusion, this is difficult to accomplish through the TLIF procedure. With respect to achieving optimal LL, insertion of hyper-lordotic angle cages is more advantageous. Cages with a $15^{\circ}$ hyper-lordotic angle resulted in greater correction of LL than cages with a $4^{\circ}$ or $8^{\circ}$ lordotic angle [6]. To achieve greater correction of $\mathrm{LL}$, the rods should be bent close to the normal lumbar lordotic angle to be corrected. If it is anticipated that the TLIF procedure will be insufficient to restore LL, anterior lumbar interbody fusion, especially at the L5-S1 segment, is an option.

In this study, LL and SVA improved more when fusion was performed at the L4-5 and L5-S1 segments than at the L3-4 segment. These findings contrast with those of Cho et al. [8]. Their study showed that the restoration of sagittal imbalance predominantly occurred when fusion was performed at the L3-L5 segment. No such restoration was observed after fusion was performed with PLIF at the L5-S1 or L4-S1 segment. One could thus infer that 
Cho et al. [8] used a cage with a $4^{\circ}$ lordotic angle, which led to less correction of LL at the L5-S1 segment. Since LL at the L4-5 and L5-S1 segments accounts for $70 \%$ of LL, the correction of LL at the lower spine has a greater effect on spino-pelvic alignment than correction at upper lumbar segments $[2,5]$. Therefore, LL should be much better corrected at the L4-5 or L5-S1 segment. A cage with a $4^{\circ}$ lordotic angle is not recommended for fusion at a lower lumbar segment. Correction of LL at the caudal segment is more effective at achieving SVA because of a leverage effect. Depending on the shape and orientation of the lumbosacral junction, the correction of LL when performing fusion at the L4-5 or L5-S1 segment is more likely to improve SVA [8].

Radiological spino-pelvic parameters related to clinical outcomes after spinal fusion surgery can include SVA, PT, and PI-LL. Increased SVA is correlated with residual pain and poor HRQOL. High PT reflects pelvic retroversion to compensate for sagittal imbalance, conferring a higher likelihood of increased postoperative pain. Therefore, decreasing the PT following short lumbar fusion for degenerative spinal diseases is required to achieve good VAS and ODI scores [7,23]. In the current study, ODI was significantly correlated with SVA, but NRS was not. LL, PT, and PI-LL turned out not to be correlated with the improvement of ODI and NRS.

Another finding in this study was that the lumbar curvature changed from straight to a harmonious lordotic curve even with short-segment fusion surgery. Adequate correction of LL after spinal fusion is described as a lordotic lumbar curvature. A harmonious lordotic lumbar curve appears to be more advantageous for maintaining sagittal balance and reducing stress at the adjacent segments. After one- to three-segment fusion, not only did sagittal alignment improve, but the lumbar spine was also restored from the straight curvature to a normal lordotic curve (Fig. 1).

This study has several limitations. First, this was a retrospective study involving the selection of a different lumbar fusion level. Second, there may have been differences in the patients with respect to the compensatory mechanism due to variation in degeneration and flexibility. Finally, division of the small number of patients unevenly into groups may have resulted in inaccurate comparisons and statistical error. Therefore, there is a need for prospective comparative studies with a longer follow-up period.

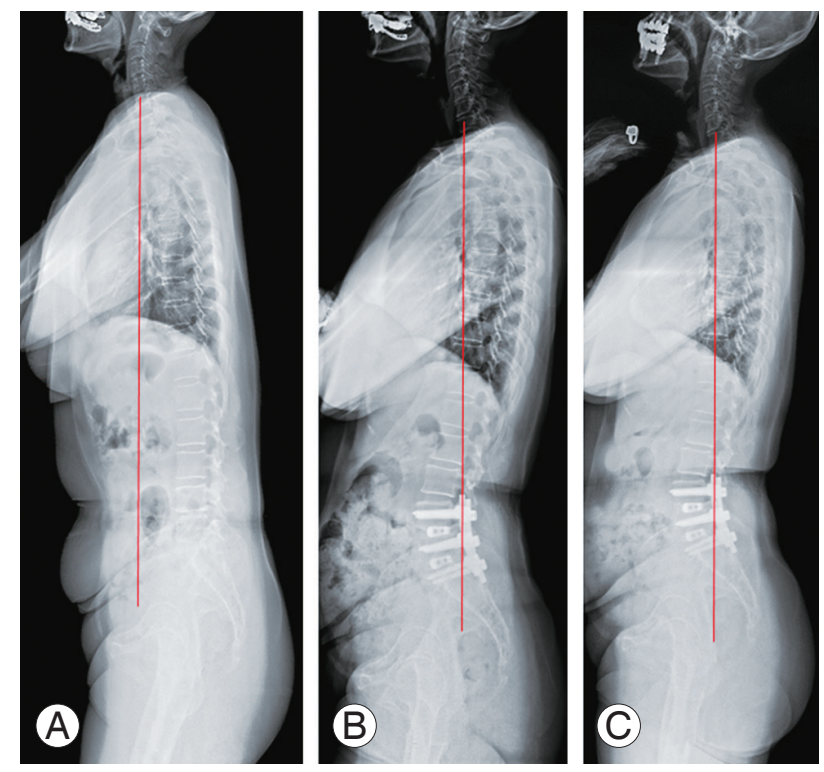

Fig. 1. A 65-year-old woman underwent transforaminal lumbar interbody fusion at L4-S1 using hyper-lordotic angle cages. Lumbar lordosis was $16.7^{\circ}$ before surgery (A), $36.5^{\circ} 1$ year after surgery (B), and $30.9^{\circ} 2$ years after surgery (C). Sagittal vertical axis was restored from $75.9 \mathrm{~mm}$ before surgery, $3.2 \mathrm{~mm} 1$ year after surgery, and $2.5 \mathrm{~mm} 2$ years after surgery. The lumbar curvature was restored from a straight curvature to a normal lordotic curve after surgery.

\section{Conclusions}

This study evaluated the change of sagittal alignment and clinical outcomes in patients with degenerative spinal disease after short-segment lumbar fusion. Short-segment lumbar fusion using hyper-lordotic cages improved LL and SVA in two- and three-segment fusion, but not in one-segment fusion. The correlations between the corrections of LL and SVA were significant in all segment fusions, especially at the L4-5 and L5-S1 segments. Postoperative improvement of SVA was well correlated with good clinical outcomes, suggesting the benefit of achieving greater correction of LL even in short-segment fusion.

\section{Conflict of Interest}

No potential conflict of interest relevant to this article was reported.

\section{Acknowledgments}

This study was supported by INHA University Research Grant (63153-01). 


\section{References}

1. Makhni MC, Shillingford JN, Laratta JL, Hyun SJ, Kim YJ. Restoration of sagittal balance in spinal deformity surgery. J Korean Neurosurg Soc 2018;61:167-79.

2. Radovanovic I, Urquhart JC, Ganapathy V, et al. Influence of postoperative sagittal balance and spinopelvic parameters on the outcome of patients surgically treated for degenerative lumbar spondylolisthesis. J Neurosurg Spine 2017;26:448-53.

3. Glassman SD, Bridwell K, Dimar JR, Horton W, Berven S, Schwab F. The impact of positive sagittal balance in adult spinal deformity. Spine (Phila Pa 1976) 2005;30:2024-9.

4. Labelle H, Roussouly P, Berthonnaud E, et al. Spondylolisthesis, pelvic incidence, and spinopelvic balance: a correlation study. Spine (Phila Pa 1976) 2004;29:2049-54.

5. Cho KJ, Kim KT, Kim WJ, et al. Pedicle subtraction osteotomy in elderly patients with degenerative sagittal imbalance. Spine (Phila Pa 1976) 2013;38:E15616.

6. Hong TH, Cho KJ, Kim YT, Park JW, Seo BH, Kim NC. Does lordotic angle of cage determine lumbar lordosis in lumbar interbody fusion? Spine (Phila $\mathrm{Pa}$ 1976) 2017;42:E775-80.

7. Kim MK, Lee SH, Kim ES, Eoh W, Chung SS, Lee CS. The impact of sagittal balance on clinical results after posterior interbody fusion for patients with degenerative spondylolisthesis: a pilot study. BMC Musculoskelet Disord 2011;12:69.

8. Cho JH, Joo YS, Lim C, Hwang CJ, Lee DH, Lee CS. Effect of one- or two-level posterior lumbar interbody fusion on global sagittal balance. Spine J 2017;17:1794-802.

9. Alqroom R. The quest of sagittal balance parameters and clinical outcome after short segment spinal fusion. Acta Inform Med 2018;26:57-61.

10. Korovessis P, Syrimpeis V, Tsekouras V. Short lumbosacral decompression plus fixation does not change the spinopelvic balance on patients with moderate degenerative spondylolisthesis and associated spinal stenosis. Spine Deform 2019;7:346-55.

11. Liu H, Li S, Wang J, et al. An analysis of spinopelvic sagittal alignment after lumbar lordosis reconstruction for degenerative spinal diseases: how much balance can be obtained? Spine (Phila Pa 1976) 2014;39(26 Spec No.):B52-9.

12. Ames CP, Smith JS, Scheer JK, et al. Impact of spinopelvic alignment on decision making in deformity surgery in adults: a review. J Neurosurg Spine 2012;16:547-64.

13. Hresko MT, Labelle H, Roussouly P, Berthonnaud E. Classification of high-grade spondylolistheses based on pelvic version and spine balance: possible rationale for reduction. Spine (Phila Pa 1976) 2007;32:2208-13.

14. Schwab F, Patel A, Ungar B, Farcy JP, Lafage V. Adult spinal deformity: postoperative standing imbalance: how much can you tolerate?: an overview of key parameters in assessing alignment and planning corrective surgery. Spine (Phila Pa 1976) 2010;35:2224-31.

15. Gardocki RJ, Watkins RG, Williams LA. Measurements of lumbopelvic lordosis using the pelvic radius technique as it correlates with sagittal spinal balance and sacral translation. Spine J 2002;2:421-9.

16. Schwab FJ, Blondel B, Bess S, et al. Radiographical spinopelvic parameters and disability in the setting of adult spinal deformity: a prospective multicenter analysis. Spine (Phila Pa 1976) 2013;38:E803-12.

17. Le Huec JC, Faundez A, Dominguez D, Hoffmeyer $\mathrm{P}$, Aunoble S. Evidence showing the relationship between sagittal balance and clinical outcomes in surgical treatment of degenerative spinal diseases: a literature review. Int Orthop 2015;39:87-95.

18. Glassman SD, Berven S, Bridwell K, Horton W, Dimar JR. Correlation of radiographic parameters and clinical symptoms in adult scoliosis. Spine (Phila Pa 1976) 2005;30:682-8.

19. Diebo BG, Ferrero E, Lafage R, et al. Recruitment of compensatory mechanisms in sagittal spinal malalignment is age and regional deformity dependent: a full-standing axis analysis of key radiographical parameters. Spine (Phila Pa 1976) 2015;40:642-9.

20. Merrill RK, Kim JS, Leven DM, Kim JH, Cho SK. Beyond pelvic incidence-lumbar lordosis mismatch: the importance of assessing the entire spine to achieve global sagittal alignment. Global Spine J 2017;7:536-42.

21. Lafage R, Schwab F, Challier V, et al. Defining spinopelvic alignment thresholds: should operative goals in adult spinal deformity surgery account for age? Spine (Phila Pa 1976) 2016;41:62-8.

22. Akgul T, Sariyilmaz K, Korkmaz M, Ozkunt O, Kaya 
O, Dikici F. Influence of distal fusion level on sagittal spinopelvic and spinal parameters in the surgical management of adolescent idiopathic scoliosis. Asian Spine J 2018;12:147-55.
23. Lafage V, Schwab F, Patel A, Hawkinson N, Farcy JP. Pelvic tilt and truncal inclination: two key radiographic parameters in the setting of adults with spinal deformity. Spine (Phila Pa 1976) 2009;34:E599606. 